\title{
The efficacy of malnutrition tools in detecting malnutrition and predicting mortality in patients with chronic heart failure
}

\author{
S. Sze ${ }^{1,2}$, P. Pellicori ${ }^{1,3}$, J. Zhang ${ }^{1,4}$, J. Weston ${ }^{1}$ and A.L. Clark ${ }^{1}$ \\ ${ }^{1}$ Department of Cardiology, Castle Hill Hospital, Hull York Medical School (at University of Hull), Kingston upon \\ Hull, HU16 5JQ, UK, \\ ${ }^{2}$ Cardiovascular Research Centre, University of Leicester, Glenfield Hospital Groby Road, Leicester, LE3 9QP, UK, \\ ${ }^{3}$ Robertson Centre for Biostatistics \& Clinical Trials, University of Glasgow, G12 $8 Q Q$ and \\ ${ }^{4}$ Faculty of Medical Science, Anglia Ruskin University, CBI 1PT, UK
}

Malnutrition is common in patients with chronic heart failure (CHF) and is associated with adverse outcome ${ }^{(1)}$. Many malnutrition tools are available ${ }^{(1)}$, however, there is no standard way of evaluating malnutrition in patients with CHF. Our study aims to compare the prevalence of malnutrition, classification performance and prognostic significance amongst 3 simple and 3 multi-dimensional malnutrition tools in $\mathrm{CHF}$ patients.

We assessed, simultaneously: 3 simple tools (Controlling nutritional status index (CONUT); geriatric nutritional risk index (GNRI); prognostic nutritional index (PNI)) and 3 multi-dimensional tools (Malnutrition universal screening tool (MUST); mini nutritional assessment-short form (MNA-SF); subjective global assessment (SGA)) in consecutive patients with CHF attending a routine follow up visit. Since there is no "gold-standard" for evaluating malnutrition in CHF patients, for each of the malnutrition tools, we used the results of the other 5 tools to produce a standard combined index which we used as a "standard" malnutrition tool. Subjects were 'malnourished' if so identified by $\geq 3 / 5$ tools.

We studied 467 consecutive ambulatory CHF patients (67\% male, median age 76 (IQR: 69-82) years, median NT-proBNP 1156 (IQR: 469-2463) ng/L). The prevalence of any degree and moderate to severe malnutrition ranged between $6-60 \%$ and 3-9\% respectively, with CONUT classifying the highest proportion of patients as malnourished.

Compared to patients with normal nutritional status, those who are malnourished tend to be older, have worse symptoms, higher NT-proBNP, lower body mass index (BMI), lower haemoglobin (Hb) and more co-morbidities.

Of the tools, CONUT had the highest sensitivity (80\%), MNA-SF and SGA had the highest specificity ( $99 \%)$ and MNA-SF had the lowest misclassification rate $(2 \%)$ in identifying moderate to severe malnutrition as defined by the combined index.

During a median follow-up of 559 days (IQR 512-629 days), 82 (18\%) patients died. In Cox models corrected for age, Charlson score, Hb, renal function, New York Heart Association (NYHA) class, atrial fibrillation (AF) and BMI, all malnutrition tools, were independently associated with increased mortality.

A base model for predicting mortality including NYHA class, log [NT-proBNP] and AF, had a C-statistic of 0.71. Amongst simple tools: CONUT $(\mathrm{C}$-statistic $=0.75)$ and amongst multi-dimensional tools, MNA-SF $(\mathrm{C}$-statistic $=0.75)$ increased model performance most compared to base model ( $\mathrm{p}<0.05$ for all). Patients who were moderately to severely malnourished according to the MNA-SF had a 6.5 times greater mortality risk than non-malnourished patients (Figure 1).

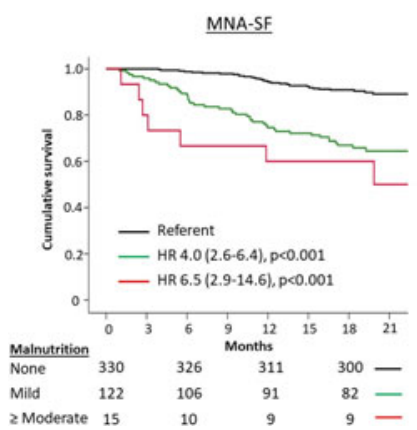

Fig. 1.

Malnutrition is common in CHF patients and is associated with increasing age, co-morbidities and HF severity. The prevalence of malnutrition is dependent on the tool used. Amongst the malnutrition tools studied, MNA-SF has the best classification performance in identifying significant malnutrition as defined by the combined index. MNA-SF also has significant prognostic value and might be appropriate for use in clinical practice when assessing patients with $\mathrm{CHF}$.

1. Lin H, Zhang H, Lin Z et al. (2016) Review of nutritional screening and assessment tools and clinical outcomes in heart failure. Heart Fail Rev 21, $549-65$. 